\title{
ARTICLE
}

\section{Clinical genomic testing: what matters to key stakeholders?}

\author{
Stephanie Best $\mathbb{D}^{1,2} \cdot$ Zornitza Stark $^{2,3,4} \cdot$ Peta Phillips $^{2} \cdot$ You Wu $\mathrm{Wu}^{5} \cdot$ Janet C. Long $\mathbb{D}^{1} \cdot$ Natalie Taylor ${ }^{6,7}$. \\ Jeffrey Braithwaite ${ }^{1} \cdot$ John Christodoulou $\mathbb{B}^{4,8} \cdot$ Ilias Goranitis $\mathbb{1}^{2,5}$
}

Received: 26 August 2019 / Revised: 2 January 2020 / Accepted: 14 January 2020 / Published online: 5 February 2020

(c) The Author(s), under exclusive licence to European Society of Human Genetics 2020

\begin{abstract}
Beyond a narrow focus on cost and outcomes, robust evidence of what is valued in genomic medicine is scarce. We gathered views on value from key stakeholders (clinical genomic staff, operational genomic staff and community representatives) in relation to three testing contexts (General Healthcare, Acute Care and Neurodevelopmental Conditions). We conducted an iterative focus group in three stages over a week using a multiphase mixed methods study, i.e. quantitative ratings and qualitative discussion. For each testing context, the characteristics of genomic testing were generated and ranked by the group using a co-productive approach. Up to 17 characteristics were identified in one scenario with several characteristics featuring in all three testing contexts. The likelihood of getting an answer was consistently reported as most highly valued, followed by the potential for the test to impact on clinical management (or wellbeing/health for Neurodevelopmental Conditions). Risk of discrimination did not feature highly across the different settings (and not at all in Acute Care). While cost was an issue in the general health setting, it was one of the least-valued characteristics in the other two testing contexts. In conclusion, co-producing an understanding of what is valued in different testing contexts, and identifying the areas of differences or commonalities, is important to maximise value provision and inform future policy to ensure that clinical genomic services meet the needs of the community and service providers.
\end{abstract}

\section{Introduction}

Supplementary information The online version of this article (https:// doi.org/10.1038/s41431-020-0576-1) contains supplementary material, which is available to authorised users.

Stephanie Best

stephanie.best@mq.edu.au

1 Australian Institute of Health Innovation, Macquarie University, Sydney, NSW, Australia

2 Australian Genomics, Murdoch Childrens Research Institute, Melbourne, VIC, Australia

3 Victorian Clinical Genetics Services, Murdoch Children's Research Institute, Melbourne, VIC, Australia

4 University of Melbourne, Melbourne, VIC, Australia

5 Health Economics Unit, Centre for Health Policy, Melbourne School of Population and Global Health, University of Melbourne, Melbourne, VIC, Australia

6 Cancer Council NSW, Woolloomooloo, Sydney, NSW, Australia

7 Faculty of Health Science, University of Sydney, Sydney, Australia

8 Murdoch Children's Research Institute, Melbourne, VIC, Australia
The momentum for the use of clinical genomic testing in mainstream clinical care, whether whole exome or genome sequencing, is gathering pace. As it does so, it is essential that we understand the complexities and opportunities that arise from early instances of implementation of genomic technologies to allow appropriate implementation strategies and policies to be designed for different contexts [1-6]. This moment in time, before genomics is commonplace, provides a unique opportunity to stop, listen and learn [7] to ensure that the services are designed and delivered in line with what service providers and consumers value.

Value is a widely debated term in healthcare, and has been associated with a move away from volume-driven service provision to linking health outcomes with cost-ofservice delivery [8]. The challenge has been ensuring value remains centred on the consumer. In part, this may be due to a lack of consensus on the meaning of 'value' and what should be prioritised [9]. We know what patients and families value is not always aligned with health providers' view [7, 10-12], which raises the importance of "patients and professionals interact(ing) as participants 
within a healthcare system in society" $[13$, p. 511$]$ to coproduce an understanding of what matters in healthcare delivery. Without a co-productive approach, knowledge is generated in silos and can hinder real-world policy development and service delivery. There is a need for service providers and consumers to work together and share responsibility for the outcomes by bringing their respective expertise to the debate.

Robust evidence of what is valued in genomic medicine is sparse. The literature offers opinions from either specific groups (e.g. oncology patients [14], or health professionals working in the cardiovascular setting [15]), or is centred on narrow viewpoints (e.g. patients' understanding of genomic results [16] or community views on genomic research [17]). Attention is often given to funding and outcomes [18, 19], with an emphasis on clinical value [20]. More rigorous studies, such as the development of the Clinician-reported Genetic testing Utility InDEx (C-Guide) [21], are needed to determine the effectiveness and the financial implications of clinical genomic testing to facilitate moving into mainstream clinical practice [22]. We also need to consider attributes that go beyond health outcomes (e.g. impact on the extended family or accessing services) and costeffectiveness of testing [23]. A recent study by Scheuner et al. [24] quantitatively explored wider stakeholder opinions on the value of outcomes from clinical genetic and genomic interventions via a two-phase survey. The researchers examined views on areas such as immediate clinical utility, as well as broader areas such as determining consanguinity and establishing ancestry. Their findings identify that stakeholders assign value to testing where the health outcome can be improved or from ending the diagnostic odyssey. We build on Scheuner et al.'s [24] findings by bringing stakeholders together to co-produce an understanding about what is of value in genomics in real-world clinical settings. The use of a co-productive approach enables debate around a consensus viewpoint rather than focusing on the views of one stakeholder group. This study goes beyond specific stakeholder interests by (i) coproducing lists of characteristics of clinical genomic testing (rather than paternity and ancestry testing) that focus group participants perceive as valuable, (ii) identifying areas of overlap between different clinical testing contexts and (iii) identifying characteristics for which value can be further explored with a larger population (using quantitative methodologies).

Access to genomic testing in Australia is currently fragmented: a range of state-based genomic health alliances along with a national health alliance are working to develop evidence to support implementation of clinical genomic testing where appropriate. The Australian Genomics Health Alliance, a national clinical and research collaboration, works with stakeholders (e.g. clinicians and consumer groups), with the aim of providing evidence for the appropriate and sustainable implementation of genomic medicine in healthcare [25]. In order to elicit what stakeholders (service providers and consumers) value from genomic testing over a range of healthcare scenarios, we chose three different clinical settings to identify if views were dependant on context. We selected a general (General Healthcare), an acute (Acute Paediatric Care [26]) and a long-term (Neurodevelopmental Conditions [27]) setting.

\section{Methods}

A multiphase mixed methods study was designed to address the research aims. We used focus groups as they encourage group interaction that can enrich the generation of information [28] and so support a co-productive approach. Focus groups provide a mechanism to uncover and appreciate participants' beliefs, understanding and thoughts around topics of interest by "co-constructing" meaning in action [29]. Central to the development and negotiation of meaning, and so the generation of research data, is social interaction [30, 31]. Tacit development of knowledge and understanding is generated through repeated interactions [32], and as a result, given the complexity of the genomics scenarios presented, we maintained a core group of participants so that they could learn to query and challenge each other, thus developing their ideas through repeated interactions. Retaining a common core of participants enabled the revealing of what participants valued, rather than seeking to identify their knowledge, expertise or understanding of clinical genomics and so fulfilled the study aim. The University of Melbourne provided ethical approval (ID 1852388 31.8.18) and Australian Genomics provided institutional approval.

\section{Participants and recruitment}

Participants were purposively sampled to secure a broad range of experience (e.g. the patient perspective of testing, clinician experience and managing testing) and promote discussion across the spectrum of testing contexts. To counter the dominant healthcare provider voice in service provision [33], we sought a majority consumer representation. Mindful of the complexities of understanding genomics, we actively recruited people with real-world knowledge or experience of clinical genomics. We sought up to 12 participants, as a maximum number to permit a single discussion group while providing a range of experiences [34], to participate in all three focus group sessions to provide a common core of participants and allow learning from the first focus group to flow into the second and third sessions. Through Australian Genomics, a national-level 
Table 1 Breakdown of attendees by representation with core noted in brackets.

\begin{tabular}{|c|c|c|c|c|}
\hline Representative group & Purpose for representative selection & $\begin{array}{l}\text { Focus group } 1 \\
\text { (General healthcare) }\end{array}$ & $\begin{array}{l}\text { Focus group } 2 \\
\text { (Acute paediatric care) }\end{array}$ & $\begin{array}{l}\text { Focus group } 3 \\
\text { (Neurodevelopmental } \\
\text { conditions) }\end{array}$ \\
\hline $\begin{array}{l}\text { Clinical genomics staff (genetic counsellor and } \\
\text { clinical geneticist) }\end{array}$ & $\begin{array}{l}\text { To provide clinician knowledge and } \\
\text { experience of genomic medicine service } \\
\text { provision }\end{array}$ & $2(2)$ & $2(2)$ & $2(2)$ \\
\hline $\begin{array}{l}\text { Operational genomics staff (managers and } \\
\text { coordinators) }\end{array}$ & $\begin{array}{l}\text { To provide knowledge and experience of } \\
\text { the complexity of genomic service delivery }\end{array}$ & $3(3)$ & $3(3)$ & $3(3)$ \\
\hline $\begin{array}{l}\text { Community representatives (advocacy group } \\
\text { representatives and people with lived experience) }\end{array}$ & $\begin{array}{l}\text { To provide experiential knowledge of } \\
\text { genomic testing }\end{array}$ & $6(4)$ & $5(4)$ & $5(4)$ \\
\hline
\end{tabular}

genomic medicine initiative [25], we identified operational genomic staff, front-line clinical staff currently using clinical genomic testing and community representatives (including Genetic Support Network Victoria, Genetic Epilepsy Team Australia (GETA), Leukodystrophy Australia, Syndromes Without A Name (SWAN) and Usher Kids Australia) or advocates with lived genomic experience to participate together. Twenty people who fulfilled the inclusion criteria were identified by the Australian Genomics Manager who invited them all to participate via email with participant information about the focus groups. Potential participants were invited to contact the lead researcher if they were interested in participating. Participants gave written consent to be audio-recorded. All the focus groups ran over one week in November 2018. In total, 11 people attended focus group 1 and ten attended focus groups 2 and 3 (see Table 1 for the breakdown of participants).

\section{Data collection tools}

A focus group protocol was developed to introduce participants, establish the expertise in the room, set out ground rules and clarify the terminology used, and then promote discussion about the characteristics of clinical genomic testing. A pilot focus group was undertaken to ensure consistency in understanding of terms used and to refine questions posed.

The focus groups were facilitated by SB and we asked participants to consider, "What are the most important things (positive and negative) for people to think about when considering genomic testing?" in relation to genomic testing itself and in relation to the three clinical settings (1st General Healthcare, 2nd Acute Paediatric Care and 3rd Neurodevelopmental Conditions). Each focus group had three phases (see Fig. 1). In Phase 1, the group identified the significant characteristics of clinical genomic testing and the key points for voting. We stimulated this discussion with the use of case studies followed with prompts informed by a pre-focus group discussion (SB, IG and EW) (see supplementary file 1). In Phase 2, we used the 'Mentimeter' app (https://www.mentimeter.com/) to prioritise characteristics

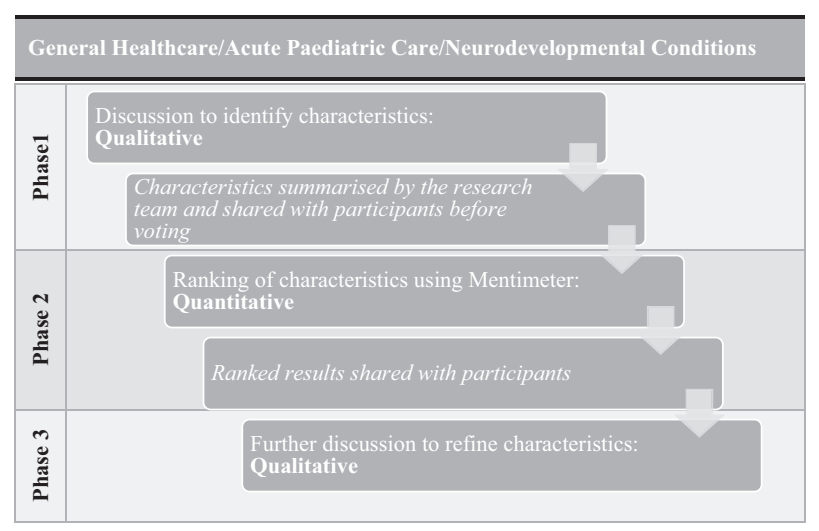

Fig. 1 Study design.

through voting, to encourage a participatory and dynamic approach to stimulate further discussion [35, 36]. Participants anonymously distributed 100 points across the characteristics they valued most highly. In Phase 3, we refined characteristics through further discussion to clarify and hone each point. The focus groups were audio-recorded, and flipchart notes were taken throughout the sessions.

\section{Data analysis}

Audio recordings were fully transcribed and imported into NVivo 11 [37] for content analysis [38]. Flipchart notes, voting results and transcribed data were used to identify broad categories (SB, EW and IG) initially independently, and followed by discussion of discrepancies to identify commonality. See supplementary file 2 for further details on data analysis.

\section{Results}

First, we present broad categories that emerged across all three focus group phases (i.e. phase 1: discussion, phase 2: voting and phase 3: refining characteristics). Then, for each health setting (i.e. general healthcare, acute paediatric care and neurodevelopmental conditions), we present a summary of findings across each focus group phase, with a 
triangulation of tabulated results across each phase according to each broad category identified.

\section{Broad categories}

To facilitate the identification of how characteristics vary across different settings (i.e. research aim ii), characteristics that participants identified were aligned to one of three categories: (1) 'context', (2) 'test' (impact and process) and (3) 'service design'. Quotes from the focus groups are provided to illustrate the categories (Key: CR-community representative, Ops-operational staff, Clin-clinician; FG-focus group 1, 2 and 3).

'Context': relates to features of the patient condition and clinical presentation. 'Test': refers to both the potential impact of clinical genomic testing and the process. For example, (1) 'test-potential impact', and (2) 'test-processes' involved in testing 'Service design': relates to features participants identified that connected to the overall delivery of genomic testing. Further examples from the transcribed focus group data for each of the broad categories can be found in supplementary file 3 . Most of the categories are found, to a greater or lesser extent, across each of the different focus group scenarios (see Tables 2-4 in Supplementary File 4).

\section{General health scenario findings}

\section{Phase 1 (Discussion) and 2 (Voting)}

Seventeen characteristics were identified (see Table 2 in Supplementary file 4), and were evenly spread across 'context', 'test' and 'service design' categories. One characteristic-likelihood of getting a genetic diagnosisstraddled both the 'context' and 'test' categories. For example, "A diagnosis, that's one of the reasons you'd consider genomic testing - is to find an answer" (CR6)FG1 is dependant on clinical presentation i.e. 'context' and also the 'test-potential impact'.

The 'context' category featured highly and included the highest-ranked characteristics: likelihood of getting a genetic diagnosis and impact on clinical management. Lowest-ranked characteristics were risk of discrimination, time to get access to the test and who has control of the genomic data that all relate to the 'service design' category.

Identifying what matters to people in terms of cost was understood to be challenging particularly in the General Health scenario "If you're asking somebody to consider a cost for something that hasn't impacted their life how could they even put a priority on it" (CR4)FG1. What cost of the test involves was debated at length, including consideration of cost to the individual, the organisation, the health system as well as inequity of access.

"Most of the time now the consultation is about the cost and who is going to pay. Not about supporting decision making, it is about the cost." (Clin1)FG1. Theme: 'service design'

Before voting, participants decided to consider cost of the test to include cost both to the individual and to funders.

\section{Phase 3 (Post voting: refined characteristics)}

Participants reported the results of the ranking process reflected the group's views and the voting process stimulated further discussion. There was concern that some characteristics overlapped others (e.g. likelihood of getting a genetic diagnosis and likelihood of a genetic basis) or were not likely to be linked with the uptake of clinical genomic testing (e.g. possibility of a retest in the future). The group debated the Phase 1 list consolidating many of the first round characteristics (e.g. in the 'context' category, severity of the condition now brought in age), narrowing the focus of others (e.g. in the 'service design' category, cost of the test (either to individual or funders) became cost of testing to you, removing the consideration of cost to funders), and adding new ones (e.g. possibility of finding out something else about yourself-test-potential impact' category). The consensus factors the group considered of value, when considering clinical genomic testing in the General Health scenario, are shown in Table 2 (Phase 3) Supplementary File 4. The final list includes 15 characteristics and retains factors relating to the 'context', 'test' and 'service design' categories.

\section{Acute paediatric care findings}

\section{Phase 1 (Discussion) and 2 (Voting)}

A more succinct list was generated for the Acute Paediatric Care scenario, (see Table 3 in Supplementary File 4). Discussion largely centred on the 'test-potential impact' category. Although the presentation of the patient was discussed it was not selected as a characteristic to vote on in Phase 2.

In total ten characteristics were identified for voting, with the highest ranked being likelihood of getting an answer, chances of changing management/treatment and chances of improving health outcomes; all related to the 'test-potential impact' category. The lowest-ranked characteristic was related to the 'service design' category e.g. cost of testing to you.

"If it was your child, I don't think you'd care about the cost." (CR5)FG2 


\section{Phase 3 (Post voting: refined characteristics)}

Participants indicated that the findings from the ranking exercise captured the group's views and prompted further debate. Following voting the group refined the wording of the characteristics and removed two characteristics (e.g. accuracy/reliability, 'test-processes' category, which they felt should be assumed features of a healthcare test), combined two (e.g. chances of changing management/treatment and chances of improving health outcomes became likely to benefit from getting a genetic diagnosis) and added one new characteristic (i.e. availability of treatment options, 'service design' category). The final list includes eight characteristics that the group believed to be important when considering clinical genomic testing in the acute paediatric care setting.

\section{Neurodevelopmental conditions findings}

\section{Phase 1 (Discussion) and 2 (Voting)}

New characteristics were identified in the Neurodevelopmental Conditions focus group. In total 14 characteristics were listed, with the 'test-potential impact' category playing a large role in the discussion around what matters to stakeholders in the Neurodevelopmental Condition setting, (see Table 4, in Supplementary File 4). The significance of well-being, rather than just health outcomes and medical treatments, featured highly in the discussion as did the broader implications of testing.

"I'd probably say [it] might not improve my physical condition but improves my mental health. I don't know what that would be - - - (CR1) Health and wellbeing" (CR10) FG3. Category: 'test-potential impact'

The highest-ranked factors included the likelihood of getting an answer, how results will impact on wellbeing/ health and ending the diagnostic journey were all linked with the 'test-potential impact' category. Lower ranked factors related to the categories 'test-processes' (e.g. time to results) and, 'service design' (e.g. risk of discrimination and who has access to data).

\section{Phase 3 (Post voting: refined characteristics)}

Discussion following the voting led to two characteristics within the 'service design' category (how results will impact access to other services and access to professional/peer support) being combined to become more inclusive as access to other services (e.g. National Disability Insurance Scheme, peer support). One additional characteristic was identified contributing to the research even if you don't get an answer and there were 15 characteristics in the final list.

In summary, there were commonalities in the findings across the three scenarios with some overlap across the different focus group testing contexts (see Fig. 2). Of note is the higher ranked characteristics were generally positive, in terms of perceived usefulness. In each setting the groups had the same priority-likelihood of getting an answerregardless of whether there are treatments available or impact on outcome. Overall, characteristics within the 'testpotential impact' category were ranked higher than the other categories. A 'service design' category prominent across the three testing contexts, was access to professional support
Fig. 2 Summary of top-ranked characteristics that are valued when contemplating clinical genomic testing by setting.

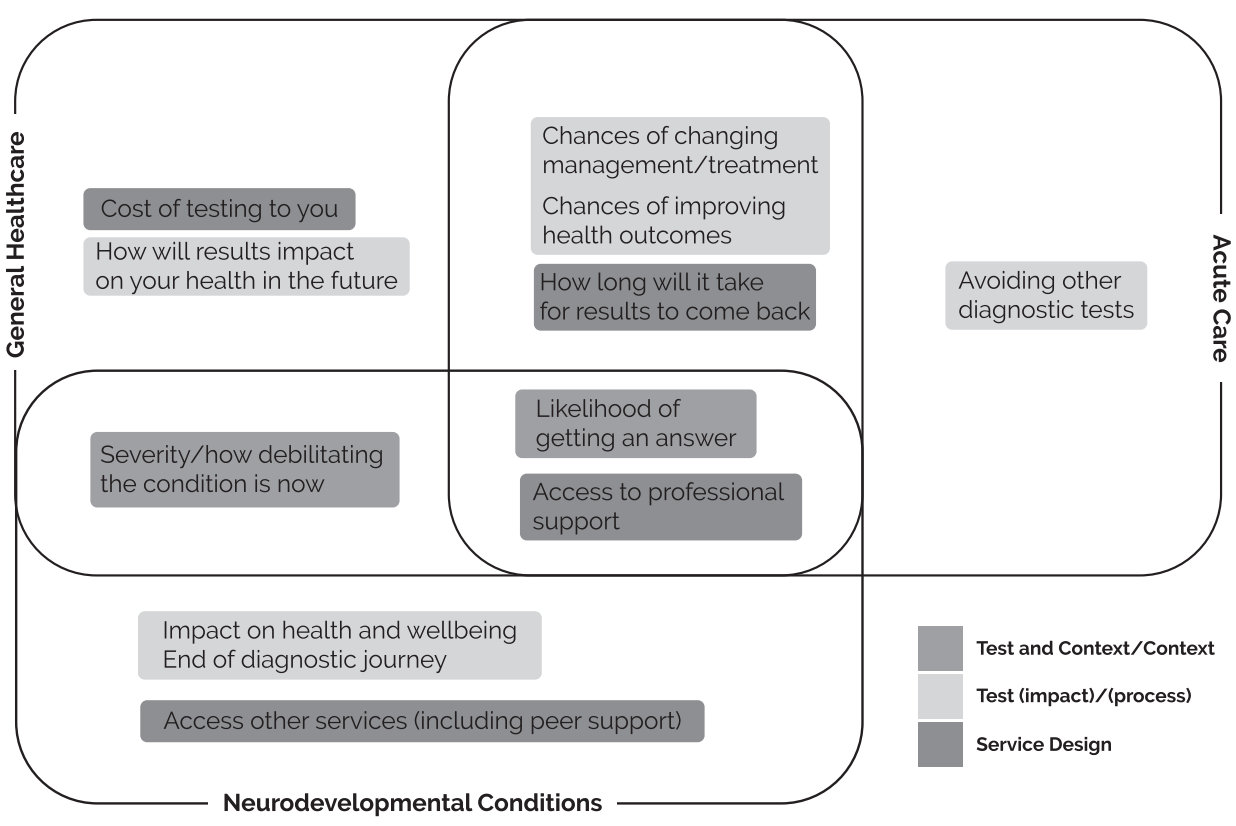


(e.g. genetic counsellors) and, for the Neurodevelopmental Conditions focus group, this was broadened to include access to peer support.

\section{Discussion}

Findings from this study provide a co-produced, clinically focused understanding of what key stakeholders value when considering clinical genomic testing in three different healthcare settings. It is important that we understand the similarities and differences across a range of clinical areas to facilitate an approach to clinical genomic testing that is tailored to each setting, and to ensure that we capitalise on the overlapping priorities to develop generalised approaches wherever possible. The highest-ranked characteristic common to all three testing contexts was likelihood of getting a diagnosis, indicating that focusing on this area should be the priority if value is to be achieved for key stakeholders. In different contexts, similar approaches could be taken to ensure that value is realised for stakeholders, for example, access to professional support ('service design' category) was highly valued across all the scenarios; for the Neurodevelopmental Conditions scenario, participants broadened this characteristic to draw on wider support including access to peers ('service design' category), valuing "social precision medicine" (CR6), reflecting the need for longer term support. Ensuring that this characteristic is acknowledged could maximise the impact for stakeholders in this clinical context. Identifying these areas allows for customisation of support without the need to reinvent what is provided, thus ensuring maximum added value across contexts.

Ranking the characteristics initially identified in Phase 1 provided an opportunity to highlight priority areas, as well as promote debate in Phase 3. Some findings were surprising when considering the relative genomic literacy in the focus group participants. For example, lower ranked areas in the General Health and Neurodevelopmental Conditions testing context included 'service design' themed characteristics (e.g. who has access to the data). Given that the focus group participants comprise people who are relatively informed about clinical genomic testing, these areas may have been expected to be ranked higher. Lower ranked priorities (e.g. risk of discrimination-'service design' category) tended to be less positive, in utility terms. This observation suggests these characteristics remain important to consider in future research activities.

Scheuner et al. [24] reported that stakeholders attribute value to clinical genomic testing when health outcomes can be improved, or the diagnostic odyssey ends. Although we find areas of overlap with Scheuner et al. [24] (see Fig. 2) we also find that stakeholder priorities shift depending on the context. For example, priorities in the Acute Paediatric
Care focus group reflected the urgency of the clinical scenario. In terms of characteristics centred on the 'testpotential impact' category (e.g. how results will impact on a child's health and wellbeing), the value adding emphasis in this clinical setting was on turnaround time. By contrast, in the Neurodevelopmental Conditions scenario, the emphasis was on lifelong disabilities with concern about the potential impact clinical genomic testing may have on accessing other services (e.g. allied health and education). Therefore, the emphasis here will be on a more holistic approach focusing on well-being in addition to health. Finally, in the General Health scenario, the presentation of the patient, i.e. 'context' category, was prominent which may impact several areas (e.g. setting up screening programmes).

Our study highlights areas of commonality between different healthcare settings, indicating areas of shared value, which provide an opportunity to generalise implementation approaches where appropriate. We also identify the importance of understanding differences in order to tailor approaches to ensure value is realised for all stakeholders. Identifying key priorities according to categories and characteristics may support organisations to focus on improving specific value for a particular condition (e.g. avoiding other diagnostic tests for acute paediatric care), or broadly concentrate on a particular area across one or more conditions (e.g. a focused approach to improve a range of 'service design', or 'test-potential impact' characteristics).

The implications of understanding where value aligns or diverges is significant for policy makers to ensure consumers receive and service providers deliver clinical genomic testing that is of value. Our research drew on innovative approaches using a co-productive research design to identify a common view of what is valued from our service providers and consumers. The use of Mentimeter not only provided an opportunity to rank priorities but, significantly, stimulated debate. This step resulted in developing conceptually clear characteristics that truly represented what focus group participants valued. Data gathered from this study can now be used to develop tools to quantify value, for example using Discrete Choice Experiment methodology [15], to further build upon these findings.

A number of limitations should be noted. All the focus groups were held in one week; this was very time consuming and potentially limited the pool of participants. On one hand, the intensive nature and short timescale of the focus groups may have permitted reflection across findings from session to session; on the other hand, it may have limited the engagement required by some to practice reflexivity from data collection to analysis. We only held one focus group for each testing context and undertaking further sessions could potentially elicit a wider range of views. We engaged people already informed about clinical genomic testing: this meant that we could not identify what 
mattered to someone new to genomics, but provided the added value that participants had an understanding of genomics. This genomic awareness did allow for in-depth debates about characteristics of significance in clinical genomic testing.

\section{Conclusion}

This is the first study to bring together a range of stakeholders, including consumers and providers, to co-produce what is of value when focusing on clinical genomic testing. We identified areas of overlap between testing contexts, providing a focus for future service provision, and thereby maximising value for all stakeholders. Genomic testing in clinical practice is at an exciting point in Australia with the Australian Government investing AUD\$500 million via the Genomics Health Futures Mission [39] and the Implementation Plan (National Health Genomics Policy Framework) providing a 'blueprint' for front-line clinical action [40]. It is essential that genomic developments are in line with stakeholders' views on what is of value to prioritise future service provision, and as such, the potential impacts of clinical genomic testing need careful consideration alongside future policy development and service design. Value in genomic medicine encompasses a wide range of components that relate to genomic testing, the clinical and the wider policy context, and determine health, non-health, and process-related outcomes for patients and their families. This study highlights that healthcare decision makers need to adopt broader value frameworks when evaluating genomic testing.

Acknowledgements We would like to thank all the focus group participants, in particular the representatives from Genetic Support Network Victoria, Genetic Epilepsy Team Australia (GETA), Leukodystrophy Australia, Syndromes Without Names (SWAN) Australia and Usher Kids Australia. This research was funded by an NHMRC Targeted Call for Research grant (GNT1113531): "Preparing Australia for Genomic Medicine". The research conducted at the Murdoch Children's Research Institute was supported by the Victorian Government's Operational Infrastructure Support Programme.

\section{Compliance with ethical standards}

Conflict of interest The authors declare that they have no conflict of interest.

Publisher's note Springer Nature remains neutral with regard to jurisdictional claims in published maps and institutional affiliations.

\section{References}

1. Manolio TA, Chisholm RL, Ozenberger B, Roden DM, Williams MS, Wilson R, et al. Implementing genomic medicine in the clinic: the future is here. Genet Med. 2013;15:258.
2. McCarthy JJ, McLeod HL, Ginsburg GS. Genomic medicine: a decade of successes, challenges, and opportunities. Sci Transl Med. 2013;5:189sr4.

3. Manolio TA, Abramowicz M, Al-Mulla F, Anderson W, Balling $\mathrm{R}$, Berger AC, et al. Global implementation of genomic medicine: we are not alone. Sci Transl Med. 2015;7:290ps13.

4. Gaff CL, Winship IM, Forrest SP, Hansen DP, Clark J, Waring $\mathrm{PM}$, et al. Preparing for genomic medicine: a real-world demonstration of health system change. npj Genom Med. 2017;2:16.

5. Taylor N, Best S, Martyn M, Long JC, North KN, Braithwaite J, et al. Combining complexity and implementation science methods to promote the uptake of genomic research into routine clinical, organisational and policy healthcare contexts across Australia: a transformative translational change programme. BMJ Open. 2018;9:E024681.

6. Stark Z, Dolman L, Manolio TA, Ozenberger B, Hill SL, Caulfied $\mathrm{MJ}$, et al. Integrating genomics into healthcare: a global responsibility. Am J Hum Genet. 2019;104:13-20.

7. Pendleton RC. We won't get value-based health care until we agree on what "value" means. Harvard Business Review. 2018. https://hbr.org/2018/02/we-wont-get-value-based-health-careuntil-we-agree-on-what-value-means. Accessed 10 Feb 2019.

8. Porter ME. What is value in health care? $\mathrm{N}$ Engl $\mathrm{J}$ Med. 2010;363:2477-81.

9. Marzorati C, Pravettoni G. Value as the key concept in the health care system: How it has influenced medical practice and clinical decision-making processes. J Multidiscip Health. 2017;10:101-6.

10. Institute of Medicine. Value in health care: accounting for cost, quality, safety, outcomes, and innovation: workshop summary. Washington, DC: The National Academies Press; 2010. https://doi.org/10.17226/12566. Accessed 10 Feb 2019.

11. Weeks JC, Catalano PJ, Cronin A, Finkelman MD, Mack JW, Keating NL, et al. Patients' expectations about effects of chemotherapy for advanced cancer. N Engl J Med. 2012; 367:1616-25.

12. Zanetti CA, Taylor N. Value Co-creation in healthcare through positive deviance. Healthcare. 2016;4.4:277-81.

13. Batalden M, Batalden P, Margolis P, Seid M, Armstrong G, Opipari-Arrigan L, et al. Coproduction of healthcare service. BMJ Qual Saf. 2016;25:509-17.

14. Halverson CME, Clift KE, McCormick JB. Was it worth it? Patients' perspectives on the perceived value of genomic-based individualized medicine. J Community Genet. 2016;7:145-52.

15. Regier DA, Weymann D, Buchanan J, Marshall DA, Wordsworth $\mathrm{S}$. Valuation of health and nonhealth outcomes from nextgeneration sequencing: approaches, challenges, and solutions. Value Health. 2018;21:1043-7.

16. Plöthner M, Schmidt K, Schips C, Damm K. Which attributes of whole genome sequencing tests are most important to the general population? Results from a German preference study. Pharmgenomics Pers Med. 2018;11:7-21.

17. Etchegary H, Green J, Dicks E, Pullman D, Street C, Parfrey P. Consulting the community: public expectations and attitudes about genetics research. Eur J Hum Genet. 2013;21:1338-43.

18. Friedman JM, Bombard Y, Cornel MC, Fernandez CV, Junker AK, Plon SE, et al. Genome-wide sequencing in acutely ill infants: genomic medicine's critical application? Genet Med. 2019;21:498-504.

19. Phillips KA, Deverka PA, Sox HC, Khoury MJ, Sandy LG, Ginsburg GS, et al. Making genomic medicine evidence-based and patient-centered: A structured review and landscape analysis of comparative effectiveness research. Genet Med. 2017;19:1081-91.

20. Grosse SD, Farnaes L. Genomic sequencing in acutely ill infants: what will it take to demonstrate clinical value? Genet Med. 2018;21:2018-20. 
21. Hayeems RZ, Luca S, Ungar WJ, Bhatt A, Chad L, Pullenayegum $\mathrm{E}$, et al. The development of the Clinician-reported Genetic testing Utility InDEx (C-GUIDE): a novel strategy for measuring the clinical utility of genetic testing. Genet Med. 2019;31:1.

22. Schwarze K, Buchanan J, Taylor JC, Wordsworth S. Are wholeexome and whole-genome sequencing approaches cost-effective? A systematic review of the literature. Genet Med. 2018;20: 1122-30.

23. Grosse SD, Wordsworth S, Payne K. Economic methods for valuing the outcomes of genetic testing: beyond cost-effectiveness analysis. Genet Med. 2008;10:648-54.

24. Scheuner MT, Russell MM, Chanfreau-Coffinier C, Peredo J, Yano EM, Hamilton AB, et al. Stakeholders' views on the value of outcomes from clinical genetic and genomic interventions. Genet Med. 2019;21:1371.

25. Stark Z, Boughtwood T, Phillips P, Christodoulou J, Hansen DP, Braithwaite J, et al. Australian genomics: a federated model for integrating genomics into healthcare. Am J Hum Genet. 2019;105:7-14.

26. Stark Z, Schofield D, Martyn M, Rynehart L, Shrestha R, Alam K, et al. Does genomic sequencing early in the diagnostic trajectory make a difference? A follow-up study of clinical outcomes and cost-effectiveness. Genet Med. 2019;21:173.

27. Australian Genomics. 2018. https://www.australiangenomics.org. au/our-research/rare-disease-flagships/\#program-1318. Accessed $24 \mathrm{Feb} 2019$.

28. Kamberelis G, Dimitriadis G. Focus groups: strategic articulations of pedagogy, politics and inquiry. In: Denzin NK, Lincoln Y, editors. Sage handbook of qualitative research. 3rd ed. London, UK: Sage; 2005. p. 887-909.

29. Wilkinson S. Focus groups in health research: exploring the meanings of health and illness. J Health Psychol. 1998; 3:329-48.
30. Kitzinger J. The methodology of focus groups. In: Seale C, editor. Social research methods: a reader. London: Routledge; 2004. p. 269-72.

31. Kristiansen TM, Grønkjær M. Focus groups as social arenas for the negotiation of normativity. Int $\mathrm{J}$ Qualitative Methods. 2017; 17:1609406917747393.

32. Wibeck V, Dahlgren M, Öberg G. Learning in focus groups: an analytical dimension for enhancing focus group research. Qualitative Res. 2007;7:249-67.

33. Carter DJ, Brown L, Saunders C. The patient's voice: australian health care quality and safety regulation from the perspective of the public. J law Med. 2018;25:408-28.

34. Robson C. Interviews and focus groups. In: Real World Research. 3rd ed. (Chichester, West Sussex: Wiley; 2011), pp. 293-300.

35. Luchenski S, Clint S, Aldridge R, Hayward A, Maguire N, Story $\mathrm{A}$, et al. Involving people with lived experience of homelessness in electronic health records research. Int $\mathrm{J}$ Popul Data Sci. 2017;1:295. https://doi.org/10.23889/ijpds.v1i1.315.

36. Nussbeck S, Rabone M, Benson E, Droege G, Mackenzie-Dodds $\mathrm{J}$, Lawlor R. Life in data. outcome of a multi-disciplinary, interactive biobanking conference session on sample data. Biopreserv Biobank 2016;14:56-64.

37. QSR International Pty Ltd. NVivo qualitative data analysis software; Version 11. QSR International Pty Ltd.; 2016.

38. Hsieh HF, Shannon SE. Three approaches to qualitative content analysis. Qualitative Health Res. 2005;15:1277-88.

39. Department of Parliamentary Services Budget review 2018-2019. Parliament of Australia. 2018. http://apo.org.au/system/files/ 173131/apo-nid173131-776116.pdf. Accessed 22 Feb 2019.

40. Commonwealth of Australia. National Health Genomics Implementation Plan. 2018. https://www.coaghealthcouncil.gov.au/ Portals/0/Genomics\%20Policy\%20Framework\%20Implementa tion.pdf. Accessed 22 Feb 2019. 